\title{
Verrucous carcinoma: a clinical case report and a review of treatment methods
}

\author{
Pavel Stanimirov ${ }^{1}$, Samuil Dzhenkov ${ }^{2}$ \\ ${ }^{1}$ Assoc. prof. dept. of Dental, oral and maxillofacial surgery, Faculty of Dental medicine, Medical \\ University- Sofia
}

${ }^{2}$ Student, Faculty of Dental medicine, Medical University- Sofia

\begin{abstract}
Verrucous carcinoma (VC) is a rare pathological finding. The etiology of the disease remains unclear. Smoking, alcohol consumption, human papilloma virus (HPV), poor oral hygiene are considered the main etiologic factors. It affects people at the age between 50 and 80 -year-old with a slight male prevalence. Once developed, the tumor grows slowly and might be wrongly taken as a benign neoplasm. However, it can be locally destructive and invasive. The most effective method of treatment is a total surgical removal of the tumor. In the following we present a clinical case report of a 46-year-old female with VC.
\end{abstract}

\section{Introduction}

Verrucous carcinoma (VC), also known as Ackermann's tumor, is a rare histological subtype of squamouscell carcinoma (SCC). It is described for a first time in 1941 by Friedel and Rosenthal [[1]]. Later, Ackermann [[1]] describes the tumor's biological behavior and the clinical findings. Considering this, he summarizes the conception of the disease as local invasive and non-metastazing.

The etiology still remains unclear. Human papilloma virus (HPV), smoking and alcohol consumption, poor oral hygiene are consider the main etiologic factors [[2]], [[3]]. The tumor affects males more often than not. Almost all of the patients are between 50 and 80 years old [1]. Krishan et al. [[4]] carried out a research with 646 patients with oral cancer, with fifty- two of them diagnosed with verrucous carcinoma.

The disease is presented clinically as a slow growing, red to white- grayish exophytic formation with a papillomatous surface, usually non- ulcerated [[5]], with well- defined margins. On palpation it appears to be soft. The histopathological examination reveals a proliferation of high-differentiated squamous epithelial cells, which are connected to the well-formed basal membrane through multiple hemidesmosomes [[6]]. Many authors describe this basal membrane as a pseudo physiological barrier of the immune system against the pathological process. The membrane's structure induces a specific "pushing"- pattern growth of the tumor rather pushes the subepithelial connective tissue layers than invade them [[7]]. These ultrastructural characteristics determine the tumor's relatively "benign" biological behavior than the other histological subtypes of the squamous-cell carcinoma [[8]]. A hybrid variant of the VC also has been described- it comprises a combination of a VC and a conventional SCC [[9]].

It is accepted that the VC should be treated just like the conventional SCC [[4]]. The surgery is still considered the most effective method of treatment of VC [[5]]. The radiotherapy is contraindicated as a definitive method of treatment of $\mathrm{VC}$ because of its ability to induce an anaplastic transformation into a conventional SCC [[10]].

\section{Case Report}

A 46-year-old patient was admitted to our clinic of oral and maxillofacial surgery in March, 2019, complaining of a painless exophytic formation on the tongue. The patient reported a heat burning of the tongue 4 years ago followed by paresthesia. A few months later a slow growing exophytic formation on the midline line of the dorsal surface of the tongue appeared. No specific treatment has been conducted since 
now. On the clinical examination we observed an exophytic, pink, non- bleeding and non- ulcerated mass with a papillomatous surface and size of $20 / 25 \mathrm{~mm}$ (Fig. 1). On palpation the tumor mass was soft, painless and well- defined. The mobility of the tongue was not affected, however it had a fissured surface, resembling the condition "lingua geographica". The patient partially edentulous, poor oral hygiene was observed too. Under local anesthesia, the tumor was totally removed by a specific hemiglossectomy. The tumor was excised together with $\mathrm{m}$. longitudinalis superior to achieve the $3 \mathrm{D}$ clear resection margins.

Histopathologically the examination revealed typical VC components together with foci of a conventional SCC. The VC component was presented as a papillary proliferation of well differentiated squamous epithelial cells with a high grade of keratinization. The tumor margins towards the connective tissue were described as "pushing" borders. A local lymphocytic infiltration was also observed. After detailed examination of the histopathological and clinical features, the diagnose was "verrucous carcinoma with conventional SCC foci". Long- term follow-up did not show any significant complications, local recurrence or metastasis.

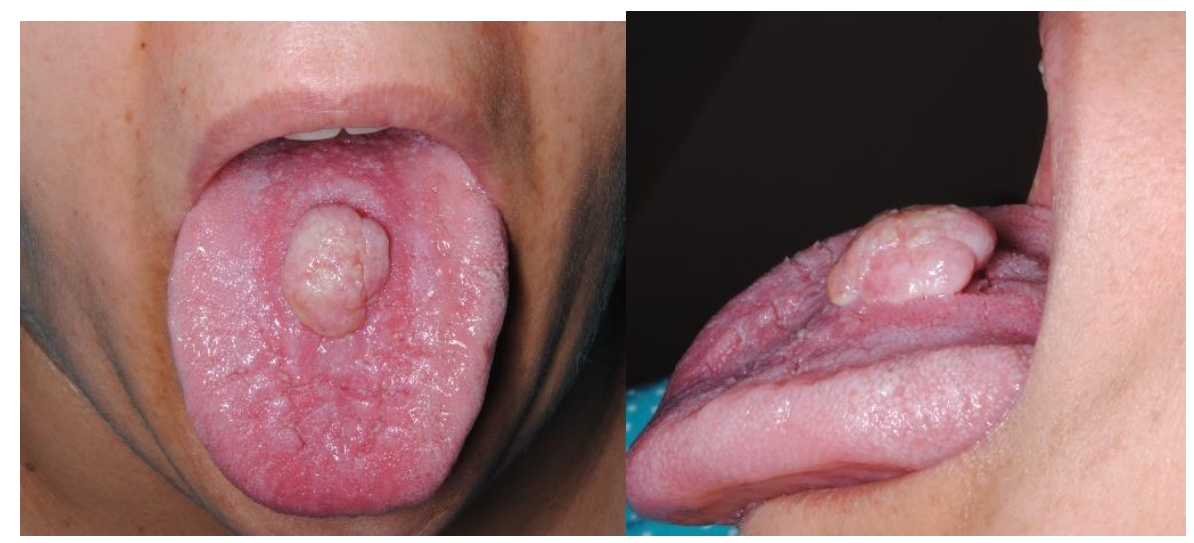

Figure 1 A, Б- An exophytic tumor mass on the dorsal surface of the tongue, pink, non-ulcerated, papillomatous surface, $20 / 25 \mathrm{~mm}$.

\section{Discussion}

VC represents a slow- growing, exophytic hyperkeratotic lesion with well- defined peripheral borders [[11]], [[12]]. Considering the clinical presentation, an appropriate diagnosis could be established followed by an adequate surgical treatment. In cases of small lesion with lack of convincing clinical signs of malignancy, an insufficient surgical removal might be undertaken. A discrepancy between the clinical and histopathological evaluation may lead to undesirable outcomes. Therefore, another surgical procedure is required in order to achieve total removal of the tumor in clear resection margins. Postoperative edema and soft tissue deformities might be related to difficulties in defining the optimal surgical lines in the re-excision required.

In the present case, the lesion is not found in the typical predilection sites for oral cancer. The clinical appearance of narrow basis exophytic growth with normal pink color and no signs of ulceration may also lead to clinical misinterpretations. However, we performed a complete surgical excision of the neoplasm. The histopathological examination revealed the presence of a hybrid variant of VC. Therefore, a surgical reexcision was performed based on the criteria for removal of conventional SCC in the oral cavity.

In all cases suspected for VC, we strongly recommend a thorough histopathological evaluation in order identify the presence of a conventional SCC foci. On the other hand, the clinical examination of the cervical area for the exclusion of neck metastases coexistence, as well as a CT scan, are of a great importance for further procedures.

The pushing pattern of growth toward the submucosal space, as well as the papillary proliferation are common microscopic findings in VC. Due to an insufficient histopathological analysis the mass can be wrongly interpreted as pseudoepitheliomatous hyperplasia or other benign epithelial lesions [[13]]. Shear and Pindborg [[14]] divided the verrucous lesions of the oral mucosa according to the histopathological findings of two groups- VC and verrucous hyperplasia. The only difference in the histologic appearance is 
the papillary proliferation in the sub epithelial layers found in the VC. The two groups of lesions are clinically indistinguishable and might even be found coexistent in some patients. Therefore, in all cases of $\mathrm{VC}$ a careful examination of the whole tumor material is required in order to exclude a coexistence with conventional SCC. When a hybrid variant is identified, the patient should be treated as with conventional SCC [[15]]. Regardless of the histopathological appearance, achievement of microscopically clear resection margins is mandatory [[16]]. The tumor is described often as more "benign" than the other histopathological subtypes of SCC due to its "pushing" pattern of growth a highly differentiated cells. However, it can also exhibit a destructive and invasive biological behavior locally [[17]].

In our previous study of 980 cases for the period 1990-2003, 28 patients (2.9\%) were reported with VC. Revision of the biopsies has been conducted in 22 out of the 28 cases. In 14 of them a typical verrucous carcinoma was found, while in the other 8- a conventional SCC coexistence was noted [[18]].

VC does not show metastatic activity and no cases of distant metastases have been reported [[9]], [[19]]. However, a metastatic activity has also been reported in cases of anaplastic transformation. The surgical approach is still considered the most effective treatment of VC [[5]], [[20]]. A combination of surgery with radiotherapy or chemotherapy improves the healing results in advanced lesions [[21]]. Nowadays, the benefits of radiotherapy as a treatment method in VS are still debated. Some authors have reported a ratio of $30-50 \%$ recurrence withing the first 6 months after using radiotherapy, as much as after surgical treatment [[4]], [[22]]. Nearly 30\% of anaplastic transformation within the first 6 months have also been reported when definitive radiation therapy was applied [[23]]. Usually chemotherapy is recommended prior surgery or as a palliative treatment modality [[20]], [[24]]. The slow growth, the poor invasiveness and the common clinical appearance of a benign pathology, can lead to an inappropriate diagnosis. In poor diagnosis clear surgical margin can hardly be established. In our clinical case a surgical excision of the tumor mass was performed together with $\mathrm{m}$. longitudinalis superior for achieving clear resection borders in $3 \mathrm{D}$. According to Oliveira et al. [[25]]. VC has a high potential for a local recurrence, which requires a long-term follow-up. We believe the hybrid histological subtype of VC is a poor diagnostic sign and the disease should be treated aggressively and radically just as in conventional SCC.

\section{Conclusion}

Verrucous carcinoma is a rare disease. The clinical picture, histopathological findings and biological behavior can lead to an inaccurate diagnosis and potential complication. VC is a rare tumor and represents just a small fraction of SCC. In cases diagnosed with verrucous squamous-cell carcinoma it is always recommended to look for the presence of a component of a conventional SCC. In suspicious tumors an incision biopsy is preferable. Macrobiopsy is recommended for lesions with typical benign clinical manifestations.

\section{References}

[1] Ackerman LV: Verrucous carcinoma of the oral cavity. Surgery 23:670,1989 squamous cell carcinomas; Scand J Dent Res. 1987; 95(3): 229-49

[2] Eversole LR. Papillary lesions of the oral cavity: relationship to human papillomaviruse. J Calif Assoc 2000; 28:922-927

[3] Tornes K, Bang G, Stromme Koppang H, Pedersen KN. Oral verrucous carcinoma. Int J Oral Surg $1985 ; 14: 485-92$

[4] Nair, M. Krishnan, et al. Oral verrucous carcinoma: treatment with radiotherapy. Cancer 1988; 61(3): 458-461

[5] Alkan, Alper, et al. Oral verrucous carcinoma: a study of 12 cases." European journal of dentistry 2010; 4(2): 202

[6] Prioleau, P.G., Santa Cruz, D.J., Meyer, J.S., and Bauer, W.C. Verrucous Carcinoma, Cancer 1980; 45: 2849-2857

[7] Sciubba, James J. "Oral cancer." Am. J. Clin. Dermatol. 2001; 2(4): 239-251

[8] McNutt, N.S. Ultrastructural Comparison of the Interface Between Epithelium and the Stroma in Basal Cell Carcinoma and Control Human Skin, Lab. Invest. 1976; 35: 132

[9] Batsakis JG, et al: The pathology of head and neck tumors: verrucous carcinoma. Part 15. Head Neck Surg 1982; 5:29 
[10] Schwartz, Robert A. "Verrucous carcinoma of the skin and mucosa. J. Am. Acad. Dermatol. 1995; 32(1): 1-21..

[11] Schrader M, Laberke HG, Jahnke K. Lymphatic metastases of verrucous carcinoma (Ackerman tumor). HNO 1987; 35:27-30

[12] Jordan RC. Verrucous carcinoma of the mouth. J Can Dent Assoc 1995; 61:797-801

[13] McCoy, J. Michael, and Charles A. Waldron. Verrucous carcinoma of the oral cavity: a review of forty-nine cases. Oral Surg. , Oral Med. , Oral Pathol. 1981 52(6): 623-629

[14] Shear, M., and Pindborg, J.J.: Verrucous Hyperplasia of the Oral Mucosa, Cancer 1980; 46:18551862

[15] Proffitt SD, Spooner TR, Kosek JC. Origin of undifferentiated neoplasm from verrucous epidermal carcinoma of oral cavity following radiation. Cancer 1970; 26:389

[16] Rheingold, L. M., and Roth, L. M. Carcinoma of the skin of the foot exhibiting some verrucous features. Plast. Reconstr. Surg. 1978; 61:605-609,

[17] Mihaylova, Zornitsa, and Evgeniy Aleksiev. "Verrucous Carcinoma (AckermanS Tumor) of the Tongue-Case Report."

[18] Stanimirov P, Bobeva A. Coexistence of verrucous and squamous cell oral cancer: analysis of 29 cases. JIMAB 2004; 2:115-7

[19] Medina JE, Dichtel W, Luna MA. Verrucous-squamous carcinomas of the oral cavity. A clinicopathologic study of 104 cases. Arch Otolaryngol 1984; 110(7):437-40

[20] Mohs, Frederic E., and William J. Sahl. "Chemosurgery for verrucous carcinoma." The Journal of dermatologic surgery and oncology 1979; 5(4)1979: 302-306

[21] McClure DL, Gullane PJ, Slinger RP, Wysocki GP. Verrucous carcinoma-changing concepts in management. J Otolaryngol 1984;13:7-12

[22] Demian SDE, Sushkin PL, Echevama RA. Perineural invasion and anaplastic transformation of vermcous carcinoma. Cancer 1973; 32:395-401

[23] Fonts EA, Greenlaw RH, Rush BF, Rovin S. Verrucous squamous cell carcinoma of the oral cavity. Cancer 1969; 23:152-160

[24] Tanaka J, Yoshida K, Takahashi M, Suzuki M. A case of verrucous carcinoma of the tongue, effectively treated with preoperative chemotherapy (uft, cddp, pep) and irradiation. Gan Kagaku Ryoho 1992; 19:525-7

[25] Oliveira, Denise Tostes, et al. "Oral verrucous carcinoma: a retrospective study in São Paulo Region, Brazil." Clinical oral investigations 2006; 10(3) 205-209 